\title{
Global lunar-surface mapping experiment using the Lunar Imager/Spectrometer on SELENE
}

\author{
Junichi Haruyama $^{1}$, Tsuneo Matsunaga ${ }^{2}$, Makiko Ohtake $^{1}$, Tomokatsu Morota ${ }^{1}$, \\ Chikatoshi Honda ${ }^{1}$, Yasuhiro Yokota ${ }^{1}$, Masaya Torii ${ }^{1}$, Yoshiko Ogawa ${ }^{2}$, \\ and the LISM Working Group \\ ${ }^{1}$ Institute of Space and Astronautical Science, Japan Aerospace Exploration Agency, \\ Yoshinodai 3-1, Sagamihara City, Kanagawa 229-8510, Japan \\ ${ }^{2}$ National Institute for Environmental Studies, 16-2 Onogawa, Tsukuba City, \\ Ibaraki 305-8506, Japan
}

(Received April 2, 2007; Revised August 24, 2007; Accepted October 31, 2007; Online published April 9, 2008)

\begin{abstract}
The Moon is the nearest celestial body to the Earth. Understanding the Moon is the most important issue confronting geosciences and planetary sciences. Japan will launch the lunar polar orbiter SELENE (Kaguya) (Kato et al., 2007) in 2007 as the first mission of the Japanese long-term lunar exploration program and acquire data for scientific knowledge and possible utilization of the Moon. An optical sensing instrument called the Lunar Imager/Spectrometer (LISM) is loaded on SELENE. The LISM requirements for the SELENE project are intended to provide high-resolution digital imagery and spectroscopic data for the entire lunar surface, acquiring these data for scientific knowledge and possible utilization of the Moon. Actually, LISM was designed to include three specialized sub-instruments: a terrain camera (TC), a multi-band imager (MI), and a spectral profiler (SP). The TC is a high-resolution stereo camera with 10-m spatial resolution from a SELENE nominal altitude of $100 \mathrm{~km}$ and a stereo angle of $30^{\circ}$ to provide stereo pairs from which digital terrain models (DTMs) with a height resolution of $20 \mathrm{~m}$ or better will be produced. The MI is a multi-spectral imager with four and five color bands with $20 \mathrm{~m}$ and $60 \mathrm{~m}$ spatial resolution in visible and near-infrared ranges, which will provide data to be used to distinguish the geological units in detail. The SP is a line spectral profiler with a 400-m-wide footprint and 300 spectral bands with 6-8 $\mathrm{nm}$ spectral resolution in the visible to near-infrared ranges. The SP data will be sufficiently powerful to identify the lunar surface's mineral composition. Moreover, LISM will provide data with a spatial resolution, signal-to-noise ratio, and covered spectral range superior to that of past Earth-based and spacecraft-based observations. In addition to the hardware instrumentation, we have studied operation plans for global data acquisition within the limited total data volume allotment per day. Results show that the TC and MI can achieve global observations within the restrictions by sharing the TC and MI observation periods, adopting appropriate data compression, and executing necessary SELENE orbital plane change operations to ensure global coverage by MI. Pre-launch operation planning has resulted in possible global TC high-contrast imagery, TC stereoscopic imagery, and MI 9-band imagery in one nominal mission period. The SP will also acquire spectral line profiling data for nearly the entire lunar surface. The east-west interval of the SP strip data will be 3-4 km at the equator by the end of the mission and shorter at higher latitudes. We have proposed execution of SELENE roll cant operations three times during the nominal mission period to execute calibration site observations, and have reached agreement on this matter with the SELENE project. We present LISM global surface mapping experiments for instrumentation and operation plans. The ground processing systems and the data release plan for LISM data are discussed briefly.
\end{abstract}

Key words: Moon, camera, imagery, multi-band, spectral, stereo.

\section{Introduction}

The Moon is the nearest celestial body to the Earth. However, these two bodies exhibit totally different features: the Earth has an atmosphere, oceans, and living plants and animals, whereas the Moon appears to have ceased all internal activity and is covered by nothing but fine dust (regolith) with small to massive craters. Since long ago, the Moon has remained a most important target to be studied.

In the 1960s and 1970s, the Moon was explored using

Copyright (C) The Society of Geomagnetism and Earth, Planetary and Space Sciences (SGEPSS); The Seismological Society of Japan; The Volcanological Society of Japan; The Geodetic Society of Japan; The Japanese Society for Planetary Sciences; TERRAPUB numerous unmanned and manned missions of the United States and the Soviet Union. Remote sensing data, in-situ experiment data, and returned sample data provided by the missions were analyzed in great detail. Consequently, an excellent dataset was obtained for use in geology, geochemistry, mineralogy, petrology, chronology, and internal structure of the Moon that is unequaled by that for any planetary body other than the Earth (Hiesinger and Head, 2006). The view revealed by handling the dataset became a standard. For example, the Moon is known not to be a primitive body. Its dark areas, called mare, mainly comprise basalt; lightly colored areas called high-lands, which are mostly anorthosite, are probably related to the existence of 


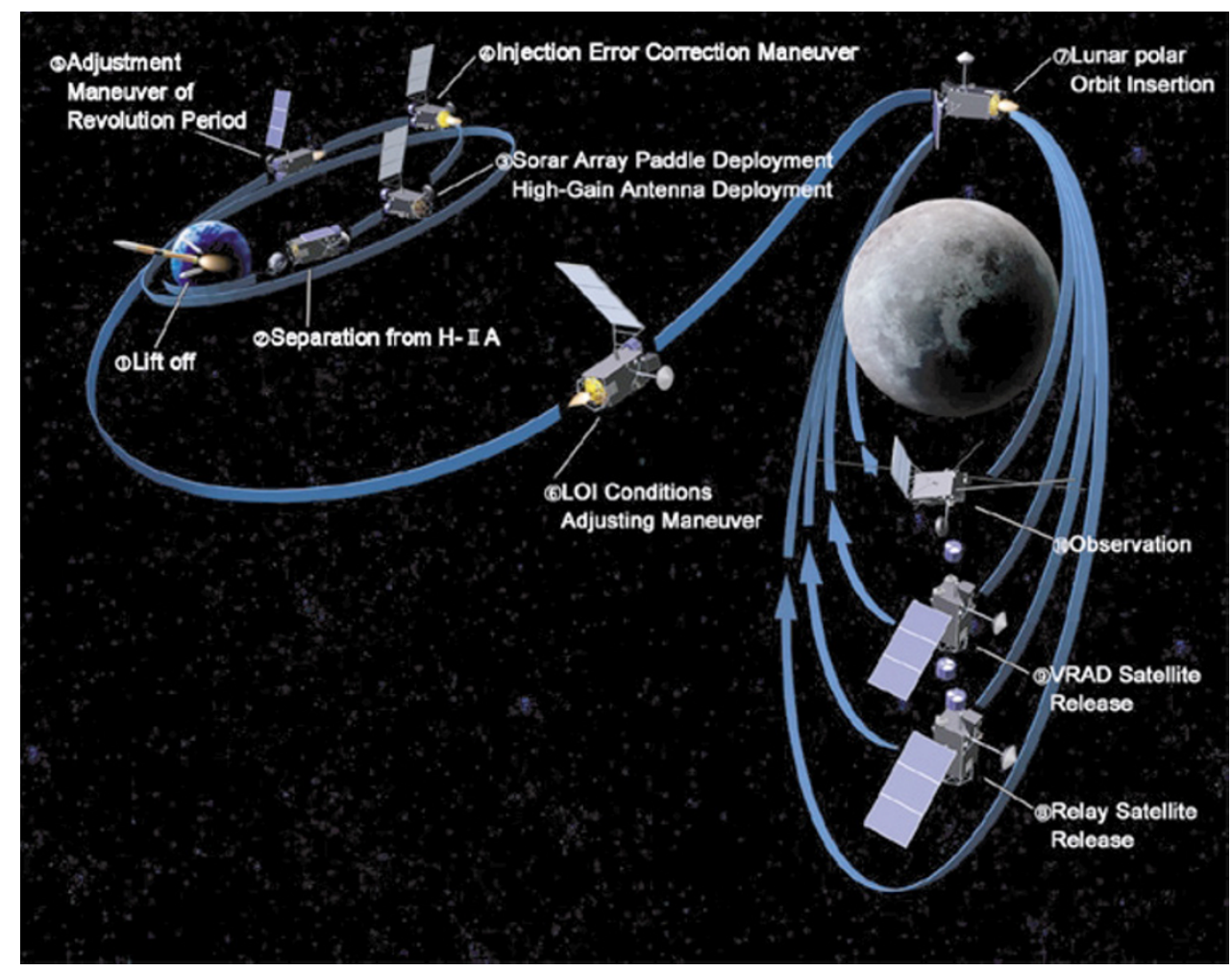

Fig. 1. SELENE mission profile (from JAXA photo archives, photo no. P-044-0001-13123). SELENE will be launched from the Tanegashima Space Center in the summer of 2007. After the launch, the SELENE will take the phasing orbit, revolving around the Earth to adjust its time-position with the Moon. After about one month of the phasing orbit, the SELENE will be injected into the orbit revolving the Moon. The initial perilune and apolune of the SELENE will be $100 \mathrm{~km}$ and $13,000 \mathrm{~km}$, respectively. During the Descending phase, the two sub-satellites will be released from the SELENE main orbiter. The SELENE main orbiter will be finally a polar circular orbit of nominal altitude of $100 \mathrm{~km}$. The LISM main power will be usually off during the phasing orbit, and occasionally on to check the power on/off with telemetry condition. First one and half months of the circulating orbit phase, the initial checkout phase in a one or a few days will be assigned for each mission instrument to confirm the possibility of transition to normal operation phase with checking if its telemetries and commands will be normally worked. In the initial checkout phase, the LISM will execute main function checks with imagery and line profiling operations.

the magma ocean. Lunar craters are mostly not of volcanic origin but are of impact origin (e.g. Melosh, 1989), mare volcanism was last active between nearly 3.9 and $3.1 \mathrm{Ga}$ (Hiesinger and Head, 2006; Head, 1976; Nyquist and Shih, 1992).

The 1990s brought a new era with new views that had been improved from previous ones, with global material mapping experiments by Galileo, Clementine, and Lunar Prospector. Using those explorers' global data, we confirmed that the Moon is more complex than had been thought. Lunar crustal terrains have been distinguished into at least three major units: (1) the Procellarum KREEP Terrane, (2) the Feldspathic High-land Terrane (FHT), and (3) the South Pole-Aitken Terrane (SPAT) (Jolliff et al., 2000). Volcanic activities likely lasted until nearly 1-2 b.y. ago (Hiesinger et al., 2000, 2003). At the lunar polar regions, the hydrogen concentration has been confirmed (Feldman et al., 1998, 1999, 2001; Lawrence et al., 2006) although whether hydrogen on the pole regions form water or not, and the origin of the hydrogen has been studied (e.g. Butler, 1997; Starukhina, 2000, 2006; Lawrence et al., 2006) but not established.

In this coming era for new views of the Moon, Japan will also launch the SELENE (Kaguya) lunar polar orbiter (Kato et al., 2007) in 2007 as the first mission of the Japanese long-term lunar exploration program, which will acquire data for scientific knowledge and possible utilization of the Moon. Three satellites constitute SELENE: the main lunar orbiter with a nominal altitude of $100 \mathrm{~km}( \pm 30 \mathrm{~km})$ (Sasaki et al., 2003) and two small sub-satellites (the relay satellite and the VRAD satellite) with a semi-major axis of $3000 \mathrm{~km}$ (Namiki et al., 1999; Iwata et al., 2007). SELENE will assume a phasing orbit after its launch and approach the Moon in about one month (see Fig. 1 for a schematic mission profile of SELENE). The key items of SELENE are listed in Table 1.

We will load an optical instrument on SELENE's main orbiter. This instrument is the Lunar Imager/SpectroMeter (LISM), which consists of three sub-instruments: a terrain camera (TC), multi-band imager (MI), and a spectral profiler (SP) (Haruyama et al., 2000, 2001, 2003a, 2007). The quality of data obtained by the LISM will be superior in spatial resolution, wavelength resolution, and signal-to-noise ratio (SNR) to that acquired in previous lunar missions. In addition, LISM will satisfy the requirements listed above. We present the LISM instruments and its operational plan for lunar global mapping.

\section{Outline of Scientific Requirements for LISM}

To acquire data for scientific knowledge and possible utilization of the Moon, LISM is expected to provide more comprehensive and detailed morphological and mineralog- 
Table 1. Key items of the SELENE main orbiter.

\begin{tabular}{ll}
\hline item & characteristics \\
\hline Launch date & Summer in 2007 \\
Launch vehicle & H2A rocket \\
Launch site & Tanegashima Space Center in Japan \\
Mission period & 1.1 years including 1.5 months for initial check out \\
Launch mass & about $1700 \mathrm{~kg}$ for dry mass \\
& about $3000 \mathrm{~kg}$ for wet mass \\
Size & $2.1 \mathrm{~m} \times 2.1 \mathrm{~m} \times 4.8 \mathrm{~m}$ \\
Consumption power & about $3000 \mathrm{~W}$ at $\beta$ (sun-moon-spacecraft)-angle of $0^{\circ}$ \\
& about $800 \mathrm{~W}$ at $\beta$-angle of $90^{\circ}$ \\
Orbit altitude & $100 \mathrm{~km} \pm 30 \mathrm{~km}$ \\
Orbit inclination & $90^{\circ}$ \\
Revolution period & $118 \mathrm{~min}$. \\
Projected speed onto the lunar surface & $1.6 \mathrm{~km} / \mathrm{sec}$ \\
Stabilization system & Three axis stabilization \\
Attitude control accuracy & $\pm 0.1^{\circ}$ in $3 \sigma$ \\
Attitude stabilization & $\pm 0.003^{\circ} / \mathrm{sec} \mathrm{in} 3 \sigma$ \\
Attitude determination & $\pm 0.025^{\circ}$ in $3 \sigma$ \\
Mission recorder & $100 \mathrm{Gbit}$ \\
Data transmission rate & $10 \mathrm{Mbps}$ \\
\hline
\end{tabular}

ical information than that obtained by past lunar explorations. We describe the outline of requirements for LISM from some important perspectives such as the age, distribution and materials of geological units to provide a scientifically important dataset.

\subsection{Age}

Understanding relative and absolute chronology is extremely important to timestamp the Moon's geologic records. The most reliable timestamp is radiometric age, as provided from rock and/or sand samples. However, global collection of samples is nearly impossible for the Moon. A popular alternative means to determine the age of a geological unit is crater counting, which is based on the simple principle that the crater size distribution in a region depends on the erupted date of the region. Correlating crater data of a region with radiometric ages from returned samples on the Moon, the crater counting chronology was developed to infer the ages of even unsampled geological units and provide the lunar stratigraphy (Hiesinger and Head, 2006; Neukum et al., 2001).

The 1990s' Galileo, Clementine, and Lunar Prospector explorers provided new perspectives of the Moon's material distribution. The cumulative crater frequency $N_{\text {cum }}(D)$ at diameter $D$ is expressed in the form of $c D^{-b}$, where $c$ and $b$ are coefficients (e.g. Melosh, 1989). Therefore, because the counting error of $N_{\text {cum }}(D)$ is estimated simply using $N_{\text {cum }}(D)^{1 / 2}$, the better the detectability of the smallest craters becomes, the less the error would be. For example, when the smallest detectable crater diameter improves by 10 times, the error would decrease to $10^{-b / 2}$. On the Moon, $b$ is 2-3.4 in the case of a crater diameter of less than $4 \mathrm{~km}$ (Melosh, 1989). Consequently, the accuracy of this case would be at least 10 times and even as much as 50 times improved when the spatial resolution increases 10 times. The smaller error for crater counting leads to improved accuracy of the model age estimation. Although the 60-100 m spatial resolution data imply a model age of $2 \mathrm{Ga}$ with nearly $1 \mathrm{Ga}$ error in $2 \sigma$ the $10 \mathrm{~m}$ resolution data can give a model age of $2 \mathrm{Ga}$ with a few tens of millions of years' error in $2 \sigma$ (Morota et al., in preparation) because past lunar mission data have not covered the entirety of mare regions at $10 \mathrm{~m}$ resolution and almost $60 \mathrm{~m}$ or worse (Wilhelms, 1987) or obtained at too much higher solar elevation angle conditions to detect craters as for Clementine (see Nozette et al., 1994 and NSSDC catalogues for information about the Clementine mission and cameras on board the Clementine spacecraft). Therefore, the $10 \mathrm{~m}$ scale global coverage with appropriate solar elevation angle conditions of less than $30-40^{\circ}$ is definitely required for imagery exploration of the Moon from the perspective of crater-counting chronology.

Another approach to investigate the relative age is specific examination of the degradation state of smaller craters of less than $1 \mathrm{~km}$ diameter. The smallest diameters of destroyed craters are nearly $1000 \mathrm{~m}, 500 \mathrm{~m}$, and $100 \mathrm{~m}$ for craters on the volcanic basalts formed in pre-Imbrian (Nectarian), Imbrian, and Eratosthenian, respectively (Wilhelms, 1987). For that reason, the degradation extents of craters with diameters of $1 \mathrm{~km}$ to $500 \mathrm{~m}, 500-100 \mathrm{~m}$, and less than $100 \mathrm{~m}$ respectively provide information about the relative age of Imbrian, Eratosthenian, and Copernican mare units in great detail. For example, an Eratosthenian unit is classified in terms of its relative age by investigating depths of craters with $400 \mathrm{~m}$ diameter in the range from $80 \mathrm{~m}$ (for fresh bowl shape craters) to $0 \mathrm{~m}$ (for destroyed craters). Therefore, data with a few tens-of-meters' or better height resolution are very useful to investigate the relative age, as inferred from the crater degradation state.

\subsection{Distribution of geological units}

Investigation of the evolutional history of the surface and subsurface is an important theme to elucidate the Moon's thermal evolution. For the first step to study the surface and subsurface, we must divide the geological units. Lunar explorations of the 1960s and 1970s acquired numerous imagery data, in which the difference of albedo, the 
Table 2. LISM (TC, MI, and SP) hardware specifications.

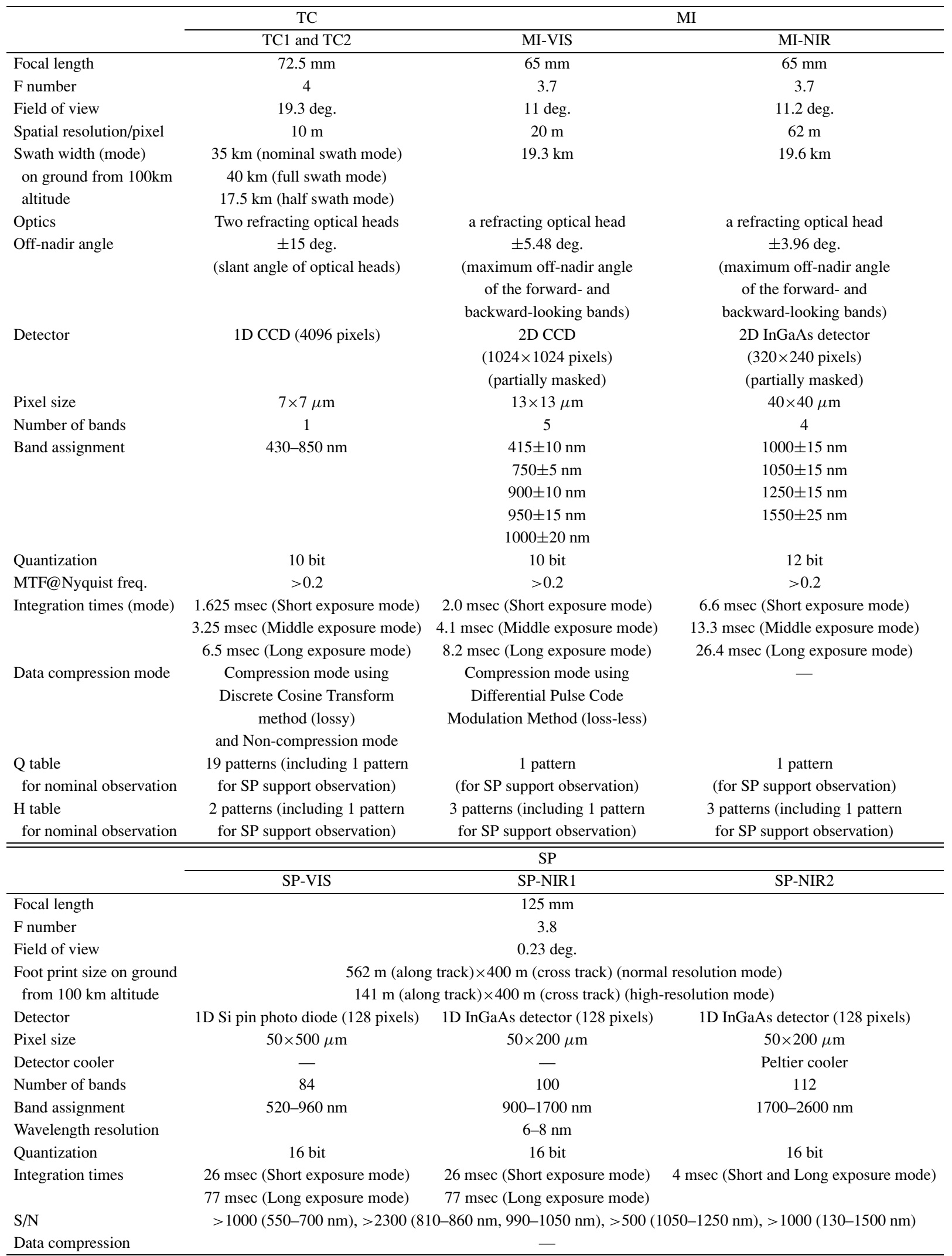

ejecta distribution, lava flow fronts and so on were obtained and became information to distinguish the geological units. Systematically acquired imagery data with a high SNR and high spatial resolution are necessary to divide the geological units in detail and exactly. For example, to distin- guish an area with $1^{\circ}$ slope area from a flat area, the SNR must be greater than 80 at the solar elevation angle of $30^{\circ}$ (Haruyama et al., 2003b). The lava flow thickness is only $20 \mathrm{~m}$ or less (Hörz et al., 1991, and their references). Consequently, $10 \mathrm{~m}$ spatial resolution or less with imaging con- 
ditions of a lower solar elevation angle of less than $45^{\circ}$ is probably necessary to provide data for spreading extents of lava flows.

Spectral information is also important for dividing the geological units. As described in the preceding subsection, especially the Clementine global multi-spectral data, with nearly $100 \mathrm{~m}$ resolution, provided information to investigate each geological unit in detail. Clementine spectral data also provided information of the Moon's subsurface structure. Tompkins and Pieters (1999) examined central peaks of more than 100 craters using Clementine UV-VIS multi-spectral data and discussed the lunar crust's compositional diversity. Pieters et al. (2001) analyzed the mineralogical diversity of the South Pole-Aitken Basin, also using Clementine UV-VIS data, and discussed implications for basin evolution. The techniques to determine the lithology of the lunar surface using Clementine UV-VIS data developed by Tompkins and Pieters (1999) and Pieters et al. (2001) have often been used (e.g. Giguere et al., 2006). Dividing geological units and analyzing the materials with multi-spectral data is now a standard method for geological study of the Moon. Clementine data were, in that sense, a key to open the new era of lunar studies. However, higher spatial resolution for multi-spectral data is now necessary to understand diverse conditions of materials, not only in the horizontal but also vertical range and to discuss them in greater detail. For example, the difference of materials between stratified lava flows under the surface, each of which is only $10 \mathrm{~m}$ thick or less (Hörz et al., 1991), might be detected using multi-band imagery with resolution greater than $100 \mathrm{~m}$ at the floor of a 100-m-diameter crater when we focus on a fresh bowl-shaped crater with a diameter-todepth ratio of 0.2 .

\subsection{Materials}

The ultimate means of studying the Moon's material, of course, is to recover samples and analyze them using state-of-the-art techniques in ground laboratories. However, sample-returning missions from all over the Moon are almost impossible. For that reason, remotely sensed data play important roles to investigate the materials constituting the Moon. In the 1990s' three missions, Galileo, Clementine, and Lunar prospector's UV to NIR camera data, in addition to data from the gamma-ray spectrometer, yielded extremely important information related to lunar crustal materials and their distributions (summarized in Jolliff et al., 2001). The information related to elements provided by the gamma-ray spectrometry and the X-ray spectrometry are important to elucidate materials of the lunar crust. On the other hand, visible to near-infrared spectral investigation has the following characteristics or merits compared to data obtained using gamma-ray and X-ray spectrometers: (1) the UV-NIR spectral observation can provide information related to the minerals themselves and (2) the spatial resolution of visible to near-infrared spectral observation onboard is $1 \mathrm{~km}$ or better currently, although the spatial resolution of gamma-ray and X-ray spectrometers onboard is a few $10 \mathrm{~km}$ at best.

The dataset of Galileo and Clementine UV-NIR multiband spectra has served important roles for lunar science progress. However, the following problems are pointed out: the dataset has a lack of bands or is insufficiently calibrated in the longer wavelength region $>1 \mu \mathrm{m}$, where key absorption bands of the lunar minerals exist. Pyroxene has its absorption bands at $0.9-1.0 \mu \mathrm{m}$ and $1.8-2.3 \mu \mathrm{m}$, olivine in $1.0-1.1 \mu \mathrm{m}$, and plagioclase in $1.2-1.3 \mu \mathrm{m}$ (e.g. Pieters, 1993). Therefore, to discuss the constituent minerals in the surface and subsurface area of the Moon in greater detail, it is necessary to acquire a comprehensive visible-to-nearinfrared spectral dataset, especially in the NIR region and well calibrated for the entire Moon.

\subsection{Summary requirements}

As described above, a more detailed and global imagery and spectral dataset is strongly required to advance the lunar sciences using an optical instrument such as LISM. Here we summarize the requirements for LISM:

- high-resolution data at $10 \mathrm{~m}$ spatial resolution at appropriate solar elevation angle conditions less than 30$40^{\circ}$;

- multi-band images with spatial resolution at $100 \mathrm{~m}$ or higher;

- stereo-pair images to produce digital terrain models (DTMs) with relative height resolution of few tens of meters; and

- spectral profiling data in the visible to the near-infrared range with onboard calibrators for wavelength and radiance.

We install LISM consisting of three sub-instruments to satisfy the above requirements: (1) Terrain Camera (TC), Multi-band Imager (MI), and Spectral Profiler (SP) on SELENE (Kaguya). The LISM (TC, MI, and SP) hardware specifications are listed in Table 2. We introduce these instruments and their operation plan in the following sections.

\section{LISM Instrumentation Outline \\ 3.1 Terrain Camera (TC)}

The mission objectives of the TC are to provide (1) global/local high-contrast mosaicked maps with $10 \mathrm{~m}$ pixel resolution of the Moon and (2) DTMs for the Moon's entirety, with relative height resolution of a few tens of meters or better and ultimately a DEM with absolute height information (Haruyama et al., 2003b, 2005, 2006a, b), which are expected to become base data for lunar sciences and future lunar explorations.

The TC has two optical heads, each of which comprises a slant refracting telescope. A linear Si-CCD detector and readout electronics are equipped in the LISM TC/MI radiation unit; an LRU (TC/MI) is attached on the $+Z$ panel of the SELENE spacecraft with the MI's two optical heads (see Figs. 2(A), 2(B), and 2(C)). The LRU (TC/MI) is covered by a box with baffles to avoid stray light problems and will be thermally controlled within $20 \pm 5^{\circ} \mathrm{C}$ with thermostats to satisfy optical performance requirements, particularly for modulated transfer function (MTF). The LRU (TC/MI) is roughly $29 \mathrm{~cm} \times 30 \mathrm{~cm} \times 22 \mathrm{~cm}$; its total weight is $9.4 \mathrm{~kg}$. The TC's two optics have identical optical layouts with a focal length of $72.5 \mathrm{~mm}$ and an $\mathrm{F}$ number of 4. The slant angles are $\pm 15^{\circ}$ from the nadir vector, or a base-to-height ratio of 0.57 . Figure 3 illustrates the stereo configuration of the TC. 
A

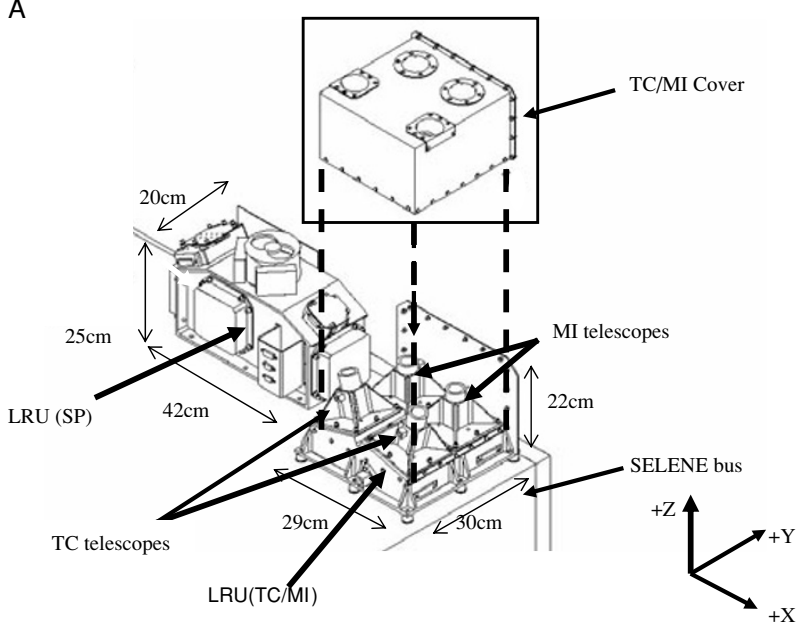

B

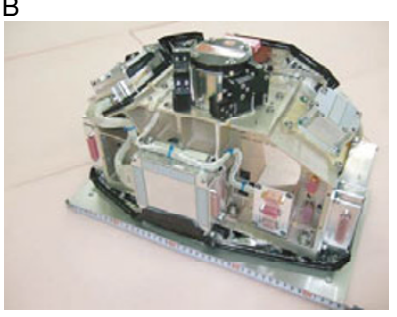

C

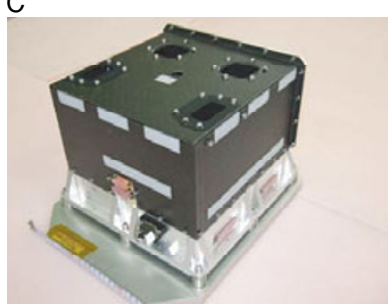

Fig. 2. (A) LISM Radiation Units: LRU (TC/MI) and LRU (SP) drawing. The LRUs are mounted on $+Z$ plane facing the Moon. Since the SELENE will be operated as the sun light irradiates onto the $-Y$ plane of the SELENE during the nominal operation, the radiators of LISM are put on the + Y plane of the LRUs. The SELENE moves along the + or $-\mathrm{X}$ direction. The reversing the flight direction will be carried out once in about half year by Yaw Around maneuver. The LRU (TC) has two slant optical heads: $\mathrm{TC} 1(+\mathrm{X})$ and $\mathrm{TC} 2(-\mathrm{X})$. The LRU (MI) also has two but nadir optical heads: MI-VIS $(+\mathrm{X})$ and MI-NIR $(-\mathrm{X})$. The LRU (TC/MI) is covered by a box. The LRUs are finally covered by black Multi-layered Insulators (not drawn in this figures). The SP NIR1 and NIR2 detectors are mounted on the $+X$ side of the LRU(SP) and the SP-VIS detector is mounted on the $-\mathrm{X}$ side. (B), (C) Photos of LISM radiator units: the left is LRU(TC/MI) and the right is LRU(SP). See text for detailed explanations.

The linear detector in each TC optical head has 4096pixels which are $7 \times 7 \mu \mathrm{m}$ with a panchromatic visible range filter of $430-850 \mathrm{~nm}$. The TC data are obtained using pushbroom scanning (see Fig. 4). Both of the TC telescopes are $72.5 \mathrm{~mm}$. For that reason, the TC cross-track field of view of each pixel is $5.5 \times 10^{-3 \circ}$. Future TCs will have $10-\mathrm{m}$ cross-track pixel resolution at $100 \mathrm{~km}$ nominal altitude. The TC sampling interval has been designed to be $6.5 \mathrm{~ms}$ and then $10 \mathrm{~m}$ on the ground along the track. Because the SELENE attitude control accuracy is designed to achieve $\pm 0.003^{\circ} / \mathrm{s}$ (or $2 \times 10^{-5 \circ} / \mathrm{s}$ ) (see Table 1 ), the dizziness of TC pointing vectors will be within $0.4 \%$ or less of TC pixel resolution for both the along and cross track fields. Therefore, a conservative evaluation for the DTM height resolution from spatial resolution over the base to height results in $17 \mathrm{~m}$. Moreover, refined stereo-matching techniques such as sub-pixel matching can achieve better height resolution than a few tens-of-meters for TC stereo pairs (Haruyama et al., in preparation). The active pixels are changed by commands in three modes: full (4096 pixels active), nominal (3504 pixels active, including four dummy pixels for compression), and half (1752 pixels active in-

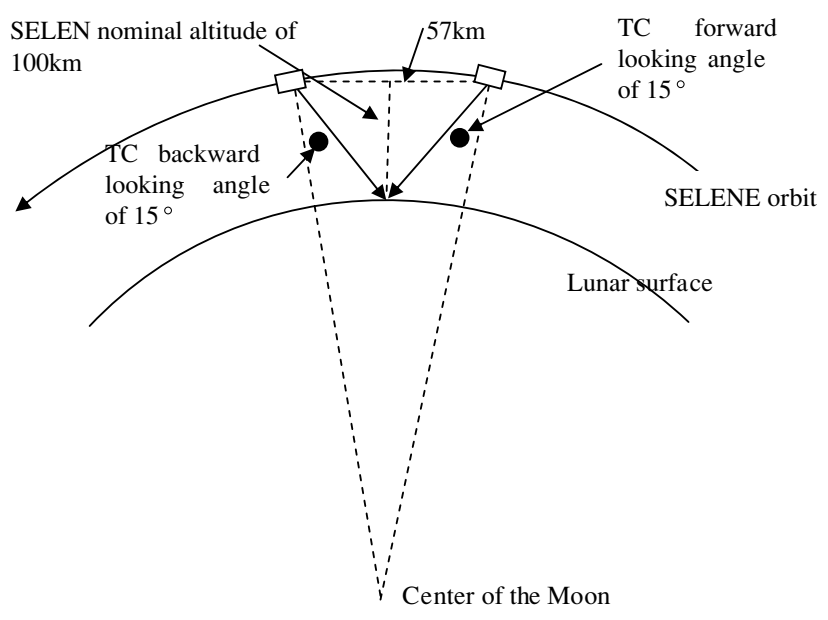

Fig. 3. TC stereo-configuration. The TC slant angles are $\pm 15^{\circ}$ from the nadir vector. When the SELENE nominal altitude is $100 \mathrm{~km}$, the base line of the TC stereo-pair is 0.57 .

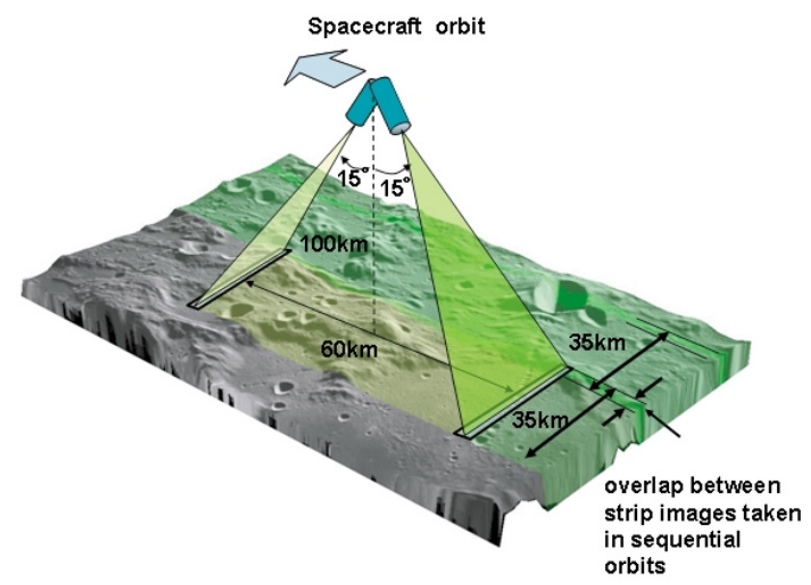

Fig. 4. TC flight configuration. The TC swath will be $35 / 40 \mathrm{~km}$ for normal/full swath mode at the SELENE nominal altitude of $100 \mathrm{~km}$, thus the TC adjacent strip images will have extended overlaps.

cluding two dummy pixels for compression). The FOV is $22.4^{\circ}, 19.3^{\circ}$, and $9.65^{\circ}$ for full, nominal, and half swath modes (the swaths on the ground will be $40 \mathrm{~km}, 35 \mathrm{~km}$ and $17.5 \mathrm{~km}$ ). The interval between SELENE's adjacent orbits will be $33 \mathrm{~km}$ at an altitude of $100 \mathrm{~km}$. Consequently, the TC adjacent strip images will have extended overlaps with the same solar angle conditions, which will facilitate the production of large mosaic images.

Data from each TC detector are read out by four lines (left-odd, left-even, right-odd, and right-even) and amplified by each amplifier. The TC analog-to-digital (A/D) conversion rate is 10 bits. The dark and gain levels of the pixel output were adjusted to a difference of less than a few DN among the pixels. The dark levels of the flight models of TC1 and TC2 have been set to 50-60 DN, depending on the exposure time mode. The exposure time will be chosen from three levels by commands: $6.5 \mathrm{~ms}$ (long), $3.25 \mathrm{~ms}$ (middle), and $1.675 \mathrm{~ms}$ (short). The saturation levels $(1023 \mathrm{DN})$ of the TC are radiance factors defined in Hapke (1993) ("reflectance" hereinafter) of $8 \%, 16 \%$, and $32 \%$ for long, middle, and short exposure times. The TC 


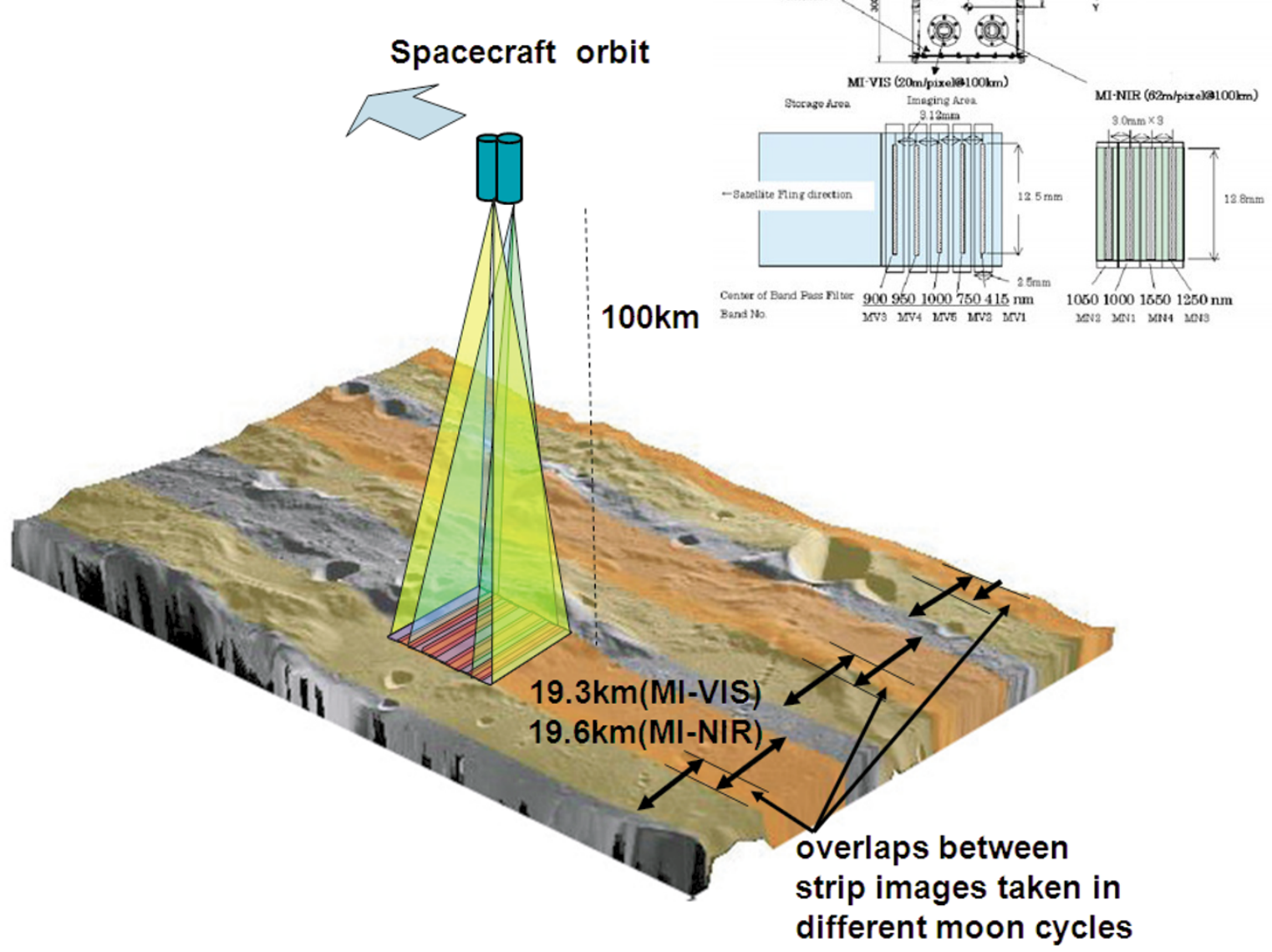

Fig. 5. MI flight configuration and its band pass filter placement. The swath of MI is $19.3 \mathrm{~km}$, and is too narrow to have overlaps between sequential strips of MI in almost areas except for the high latitudes. The imaging gaps left in the one Moon cycle operation for MI will be taken in the coming opportunities. To set the appropriate orbits for MI filling the observation gaps, the SELENE will carry out the change of its orbital plane.

flight model test revealed a high SNR of more than 100 at a solar reflectance intensity of $2 \%$, which makes it possible to distinguish one-degree slope areas from flat areas at solar elevation angles of less than $40^{\circ}$ (Haruyama et al., 2003 b, 2004). We adopted an onboard lossy DCT compression method for the TC to reduce the data volume. There are 32 patterns installed in the onboard Q-table, including 13 for redundancy. The TC has a non-compression mode. Non-compressed data will be used to estimate the compression error.

\subsection{Multi-band Imager (MI)}

The mission objectives of the MI are to provide global mosaicked maps of geological units with $20 \mathrm{~m}$ and $62 \mathrm{~m}$ pixel resolutions in the visible and near-infrared ranges, which will be one-order higher resolution than Clementine's UV-VIS and NIR camera data (Ohtake et al., 2003, 2007). The maps produced using the MI color band data will be used (1) to search for the most primitive lunar costal materials, (2) to clarify the horizontal and vertical structure of Mare basalt, (3) to survey the mantle-rock distribution, and (4) to clarify the impact crater formation mechanism.

The MI has two optical heads, MI-VIS and MI-NIR, for the visible range and near-infrared range. Each head is composed of a nadir-refracting telescope, a two-dimension Si-
CCD for MI-VIS and a two-dimension InGaAs for MI-NIR with readout electronics installed in the LISM TC/MI radiation unit, and a LRU (TC/MI) with two TC optical heads, as described in the TC section. The thermal condition of the LRU (TC/MI), within $20 \pm 5^{\circ} \mathrm{C}$, is sufficient for the MI optical performance, particularly for the SNR. The two MI optics have almost identical optical design with a focal length of $65 \mathrm{~mm}$ and $\mathrm{F}$ number of 3.7. The MI detectors are $1024 \times 1024$ pixels, with pixels of $13 \times 13 \mu \mathrm{m}$ for MIVIS and $320 \times 240$ with pixel size of $40 \times 40 \mu \mathrm{m}$ for MINIR. Five and four band-pass filters are mounted over several lines on the MI-VIS and MI-NIR detectors; the unused lines are masked to avoid stray light. The band-pass filter assignment was determined to provide mineral distribution information, as illustrated in Fig. 5. The MI band assignment (based on Ohtake et al., 2007) is presented in Fig. 6 (lower); those of Clementine's UV-VIS and NIR in Fig. 6 (upper) are based on those by Nosette et al. (1994). The MI acquires the data by push-broom scanning, as does the TC. In fact, MI-VIS/NIR will have $20 \mathrm{~m} / 62 \mathrm{~m}$ cross-track pixel resolution at a $100 \mathrm{~km}$ nominal altitude and a $13 \mathrm{~ms} / 39 \mathrm{~ms}$ (or $20 \mathrm{~m} / 60 \mathrm{~m}$ on the ground) along-track sampling interval. The active pixels of the MI are fixed at 1024 pixels (including 62 dummy pixels for compression)/320 pixels 


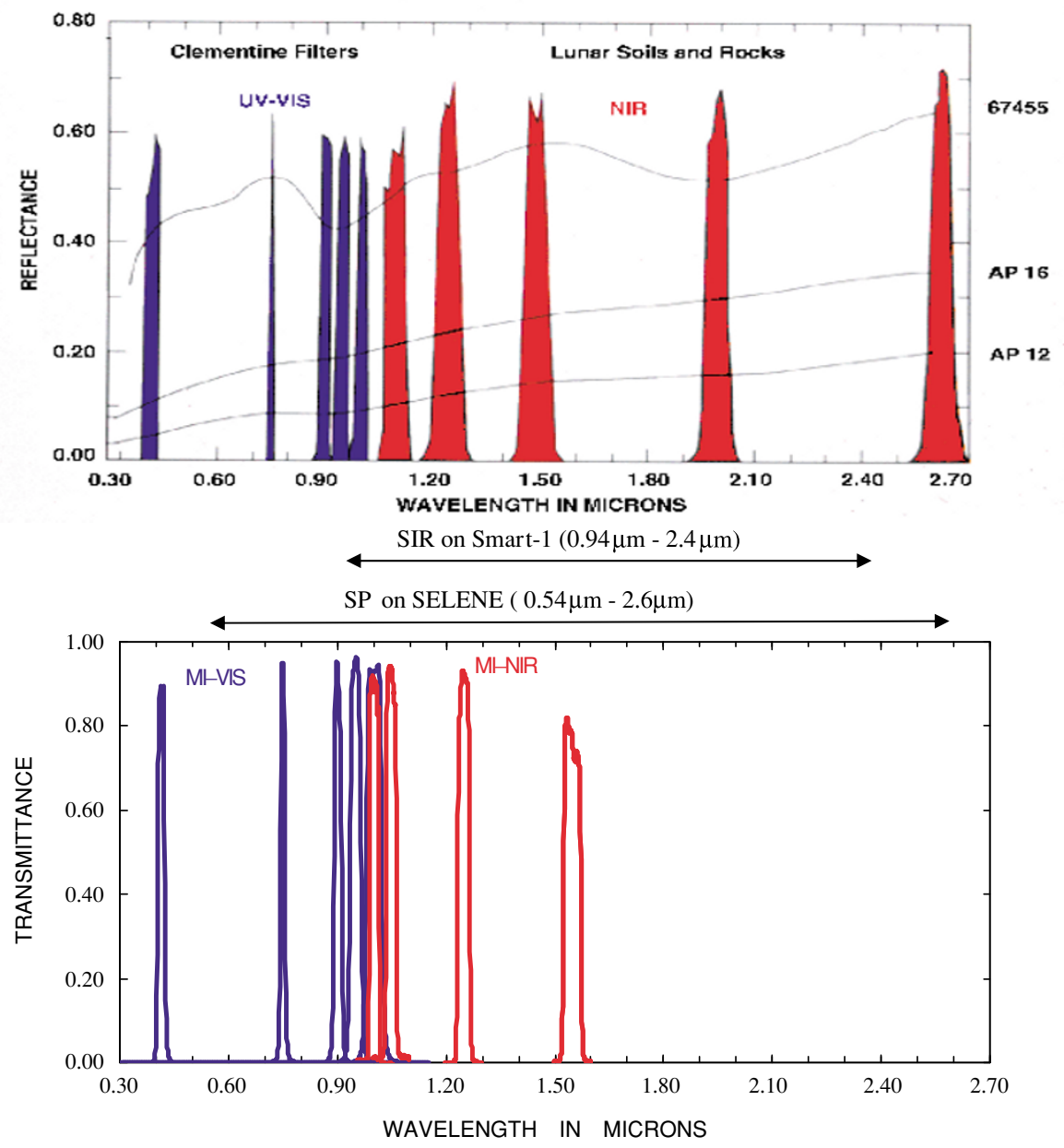

Fig. 6. Bandpasses of Clementine filters for UV-VIS and NIR cameras superimposed on reflectance spectra of lunar samples (upper: Nosette et al., 1994) and those of MI-VIS and NIR (lower) with spectral coverage of SP on SELENE and that of SIR on Smart-1 (Basilevsky et al., 2004).

(including 16 pixels for compression) for MI-VIS and MINIR. The FOV is $11^{\circ}$ for both MI-VIS and NIR; the swath on the ground will be $19.3 \mathrm{~km}$. The interval between SELENE's adjacent orbits at the equator will be $33 \mathrm{~km}$ at a nominal altitude of $100 \mathrm{~km}$. For that reason, the MI adjacent strip images will not have an extended overlap, and a few changes of SELENE's orbit plane during the mission period will be required to achieve global coverage using MI nine-band imagery.

The readout method of the MI-VIS detector is frame transfer. A frame transfer correction will be executed on the ground. In contrast, the MI-NIR detector uses an electrical shutter, which prevents ghost images resulting from frame transfer. The A/D rate is $10 \mathrm{bit} / 12$ bit for MI-VIS/NIR. The exposure time is selected by commands from three levels: $8.2 \mathrm{~ms}$ (long), $4.1 \mathrm{~ms}$ (middle), and $2.0 \mathrm{~ms}$ (short) for MI-VIS, and $26.4 \mathrm{~ms}$ (long), $13.2 \mathrm{~ms}$ (middle), and $6.6 \mathrm{~ms}$ (short) for MI-NIR. The dark levels of the MI flight model have been set at about 40 DN for MI-VIS and 200-900 DN depending on the row number of the pixels. The saturation levels 1023 DN/4095 DN of MI-VIS/NIR respectively represent solar reflectance intensities of $15 \%, 30 \%$, and $60 \%$ for long, middle, and short exposure times. The MI flight model test revealed a high SNR of greater than 100 at a solar reflectance intensity of $5 \%$. The MI-VIS data will also be compressed using the DPCM lossless compression method to reduce the data volume. Compression experiments using simulated images demonstrated a possible achievement of less than $80 \%$ (Haruyama et al., in preparation). The MINIR data are not compressed onboard because the MI-NIR data rate is about one-tenth that of MI-VIS.

\subsection{Spectral Profiler (SP)}

The primary purpose of the SP is to provide global and regional mineral/rock type distributions on the Moon by obtaining accurate spectral characteristics of the surface (Matsunaga et al., 2001, 2003). The mineral/rock information will serve as a fundamental database of each geological unit, which will be distinguished by discrete color information of the surface provided by the MI (see Fig. 7).

The SP is a Cassegrain line spectral profiler. The SP radiation unit, LRU (SP), attached on the $+Z$ panel of SELENE, consists of foreoptics and two spectrometers, VIS and NIR. The LRU (SP) is thermally controlled within $20 \pm 5^{\circ} \mathrm{C}$ with thermostats to achieve high spectral and radiometric stability. The LRU (SP) is roughly $42 \mathrm{~cm} \times 20 \mathrm{~cm} \times 25 \mathrm{~cm}$; its total weight is about $10 \mathrm{~kg}$ (see Fig. 2).

The light focused by the foreoptics is introduced to the spectrometers through a narrow slit of the SP-VIS spectrometer, which has a 1D (128 pixels) Si pinphoto-diode sensor of $50 \times 500 \mu \mathrm{m}$ mounted by a band pass filter cover- 


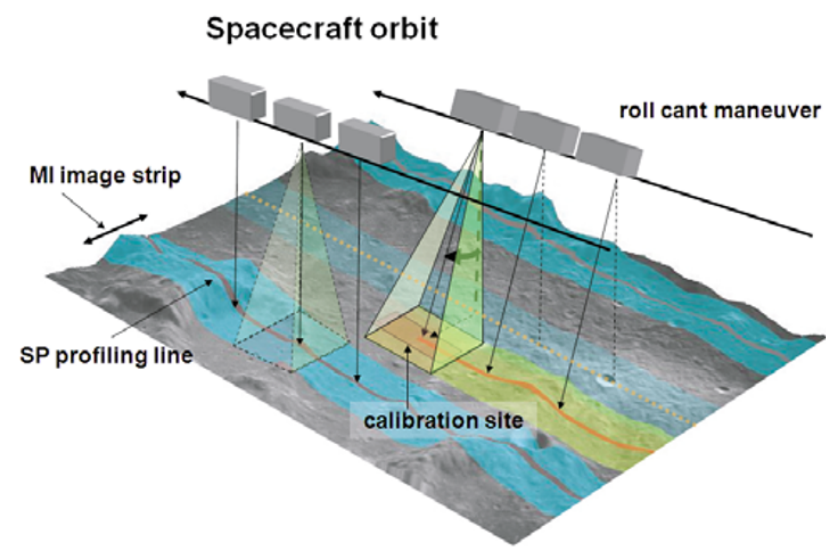

Fig. 7. SP flight configuration. The mineral distribution of the surface of the Moon will be investigated by color maps produced from MI data, and the detailed mineralogy of the units distinguished by color maps will be performed by using the SP line profiling but continuous spectral data. To observe a calibration site in appropriate illumination conditions at least a few times in the SELENE nominal mission period, roll-cant maneuvers will be carried out. An area near the Apollo 16 landing sites is a candidate of calibration site.

ing 490-980 nm, avoiding secondary diffracted light. The light is split by a dichroic mirror into NIR1 and NIR2 detectors inside the SP-NIR spectrometer. Both detectors of the SP-NIR spectrometers have the same type 1D (128 pixels) InGaAs sensor of $50 \times 200 \mu \mathrm{m}$ pixels. The band-pass filters cover 900-1700 nm for SP-NIR1 and 1700-2600 nm for SP-NIR2. The SP-NIR2 detector is cooled using a threestage Peltier cooler to $243 \mathrm{~K}$ to reduce thermal noise during operation. The spectral resolutions are $6 \mathrm{~nm}$ for SP-VIS and $8 \mathrm{~nm}$ for SP-NIR1 and NIR2. There are 84 bands for SP-VIS, 100 for NIR1, and 112 for NIR2. The wavelength coverage of the SP is wider than that of SIR on Smart-1 (see Fig. 6).

The SP will have a field of view (FOV) of $0.23^{\circ}$, or $400 \mathrm{~m}$ cross-track pixel resolution on the ground, at a $100 \mathrm{~km}$ nominal altitude. The along-track sampling interval is $90 \mathrm{~ms}$, corresponding to $562 \mathrm{~m}$ on the lunar surface. Four $90 \mathrm{~ms}$ data are integrated in the nominal resolution mode, packed, and transmitted to SELENE's data handling system. The $90 \mathrm{~ms}$ data are processed without integration in the highresolution mode. The analog-to-digital (A/D) conversion rate of the SP is 16 bit. The exposure time for SP-VIS and NIR1 will be selected from two levels: $77 \mathrm{~ms}$ (long) and $26 \mathrm{~ms}$ (short). The exposure time for SP-NIR2 is $4 \mathrm{~ms}$. The saturation levels $\left(2^{16} \mathrm{DN}\right)$ of the $\mathrm{SP}$ are solar reflectance intensities of nearly $60 \%$, and $20 \%$ for long and short exposure times. The SP flight model test yielded a good SNR of more than 1000 at 550-700 nm and 1300-1500 nm, more than 2300 at $810-860 \mathrm{~nm}$ and $990-1050 \mathrm{~nm}$, and more than 500 at $1050-1250 \mathrm{~nm}$ at a solar reflectance intensity of $6 \%$ for SP-VIS and NIR1. The SNR of SP-NIR2 was more than 300. The SP has an onboard calibrator module that consists of a lighting module attached on the LRU (SP) foreoptics and two light source (halogen lamp) modules for radiance and wavelength calibration in the data processing unit (DPU) (SP) installed in the SELENE bus component. The two modules are connected by optical fibers.

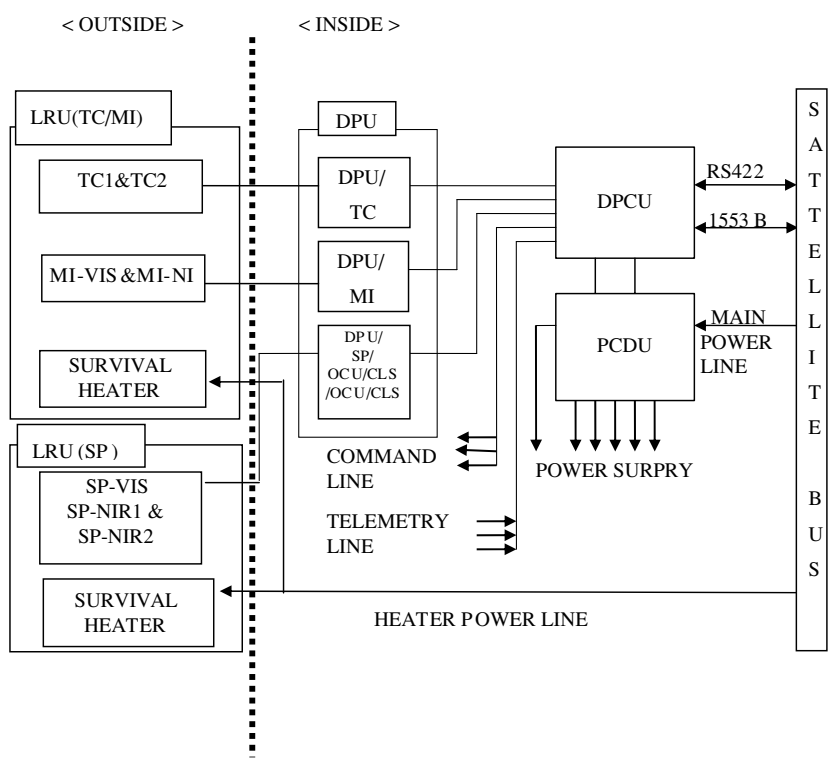

Fig. 8. Block diagram of the LISM.

\subsection{LISM block diagram}

Figure 8 presents a block diagram of LISM, which consists of five separate units: the LISM radiometer unit of TC and MI (LRU TC/MI), LISM radiometer unit of SP (LRU SP), DPU, data processing and control unit (DPCU), and the power control and distribution unit (PCDU). The LRU (TC/MI) and LRU (SP) are attached on the $+\mathrm{Z}$ plane of the SELENE bus (see Fig. 2).

Seven LISM detectors are used: two for TC (T1 and T2), two for MI (MIV and MIN), and three for SP (SP-VIS, SPNIRT, and SP-NIR2). The analog signals transferred from each LRU are amplified and converted to digital signals in the DPU. The A/D rates of the detectors are different, as described in the above sections: 10 bits for TC1, TC2, and MIV; 12 bits for MIN; and 16 bits for SP-VIS, NIR1, and NIR2. The digitized data in the DPU are transferred to the DPCU. The DPCU comprises a telemetry/command processing sub-unit, a TC/MI data processing sub-unit, an SP data processing sub-unit, and a heater control sub-unit. The TC/MI data processing sub-unit has a data-encoding chip that performs data compression for data of both the TC and MI-VIS. The MI-NIR data and the SP data are not compressed. The LISM has two interfaces with SELENE's bus system: an RS-422 I/F for high-data-rate communication and a MIL-1553B I/F for low-data-rate communication. The LISM, lunar radar sounder (LRS), and high-density television camera (HDTV) on SELENE use an RS-422 I/F line. All LISM science data are transferred via RS-422 I/F to SELENE's bus system. The data rate from LISM to the bus system is limited to $5 \mathrm{Mbps}$. A data rate limiter will operate when the LISM data rate is greater than $5 \mathrm{Mbps}$ (for example, in the uncompressed condition) and will stop transmitting the data from the LISM to the bus system. The limiter will be released immediately when the data rate is under the $5 \mathrm{Mps}$ threshold. The MIL-1553B I/F is used for communication of control commands and housekeeping telemetry data between LISM and SELENE's bus system. The telemetry/command processing sub-unit per- 


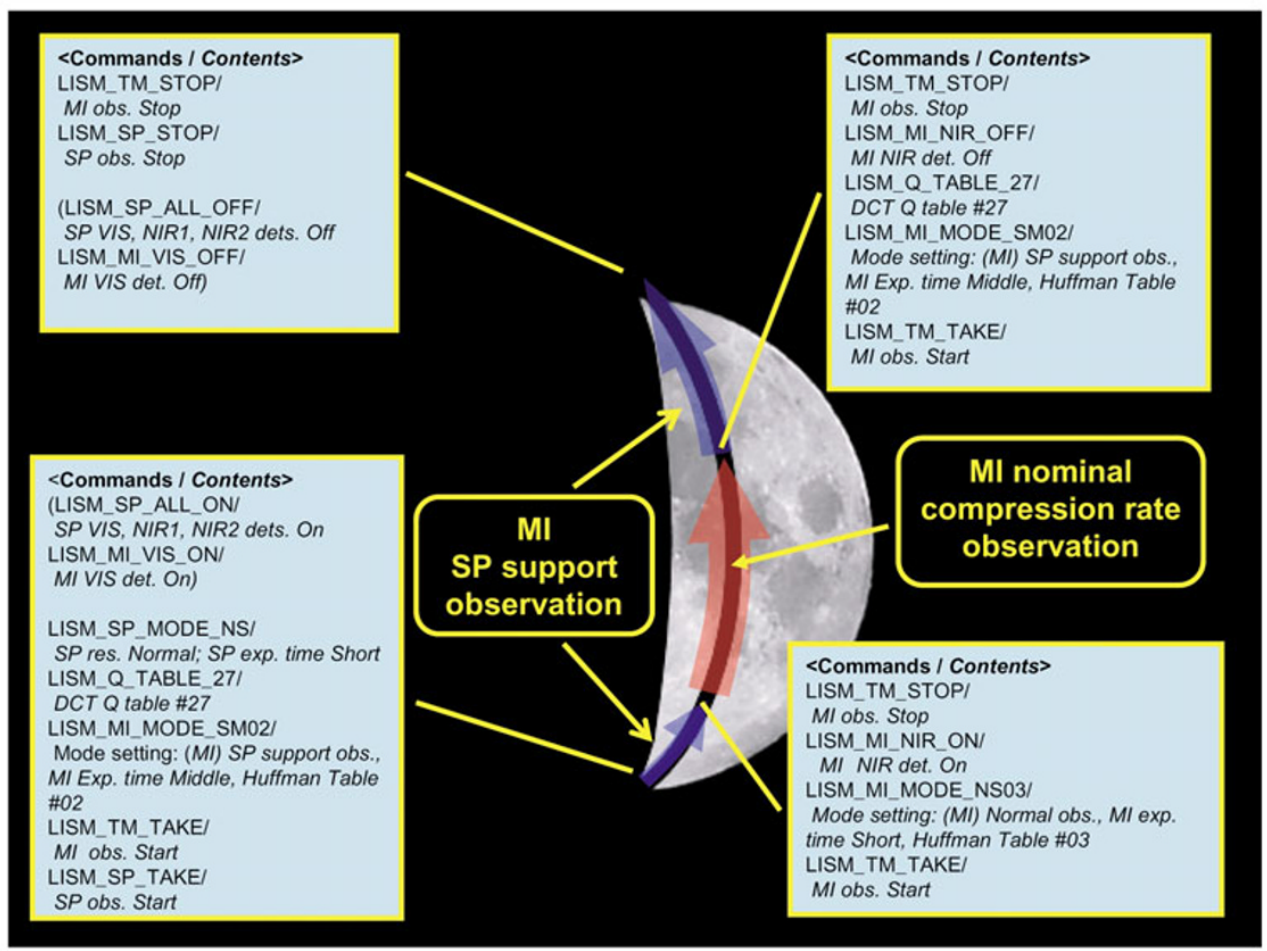

Fig. 9. Schematics of a typical revolution observation by MI: MI lower latitude observation. In this revolution, MI observes with compressing MI-VIS and NIR data by loss less DPCM method in the lower latitude and executes the SP support observation with highly compressing the MI VIS one band (usually $750 \mathrm{~nm}$ band) data in the middle and higher latitude. SP will observe through the daytime. At changes of observation type, the LISM once stops the data acquisition and change the operational detectors and reset the modes for the exposure time, and compression tables. Detectors of MI and SP will "not" be off even in the night side to maintain stable conditions.

forms command processing, telemetry data acquisition, and all instrument controls.

The SELENE bus voltage will vary from 50.9 to $52.8 \mathrm{~V}$ in the sunshine and from 32.6 to $51.2 \mathrm{~V}$ in the shade. The PCDU in LISM is a DC/DC power converter to supply stable voltage to the LISM internal units. The temperatures of the TC/MI telescopes and SP spectrometers are adjusted automatically to $20^{\circ} \mathrm{C} \pm 5^{\circ} \mathrm{C}$ by a thermal-controlled heater depending on the temperature when the LISM main power is on. The thermal control unit does not function when the LISM main power is off. Instead, the survival heaters are independently controlled by the spacecraft bus system operating to maintain the instrument temperature as higher than $-30^{\circ} \mathrm{C}$.

\section{LISM Operation Plan}

\subsection{Nominal operation}

To store data, SELENE has a mission data recorder with 100 Gbit memory. The data transmit rate from SELENE to the Usuda Deep Space Center (UDSC) in Japan is 10 Mps. Such large resources for data recording and transmission have not previously been allotted for Japanese deep space science missions. However, the data volume will exceed $1 \mathrm{Tbit} /$ day when TC and MI operate simultaneously in the daytime without data compression. For that reason, the data volume per day from LISM would be too great to transmit to the UDSC during its operative time of at most $12 \mathrm{~h}$ for a Moon orbiter. The LISM data volume was set to $50 \mathrm{Gbit} / \mathrm{day}$ after considering the total data acquisition rates of other mission instruments and bus systems. We have discussed methods to reduce the daily data volume for LISM and concluded that the following operational restraints should be imposed.

1) $\mathrm{TC}$ and MI will not operate simultaneously.

2) TC/MI will compress data on board.

3) $\mathrm{TC} / \mathrm{MI}$ will acquire images within a scheduled latitude range.

The following LISM default operation plan to achieve global coverage in one year was drawn based on the above restrictions. During the nominal SELENE mission period of one year, or 13 Moon cycles, we will perform 1) TC stereomapping in three Moon cycles, 2) TC mono-telescope mapping under illumination conditions of eastward and westward lower solar elevation angles of $60^{\circ} \mathrm{N}$ to $60^{\circ} \mathrm{S}$ in two Moon cycles, and 3) MI visible and near-infrared mapping in six Moon cycles. The TC/MI observations during one SELENE revolution around the Moon will be divided into two compression rates: nominal compression rate observations, and SP support observations with a high compression rate of about $2 \%$ to detect SP observation lines by one telescope system (TC or MI). Figure 9 presents schematic illustrations for a typical revolution operation by MI, in which MI is depicted in nominal operation mode with MI-VIS and NIR compression using the lossless DPCM method in the lower latitudes and SP supports observations with highly compressed MI VIS one-band (typically the $750 \mathrm{~nm}$ band) data in the middle and higher latitudes. The nominal compression rate will be less than $30 \%$ for TC and $80 \%$ for MI. Therefore, we will have achieved global data acquisition of 
two TC high-contrast images, one stereo-pair image, and a set of MI 9-band images by the end of the mission. The SP will acquire data throughout the daytime without data compression. The east-west interval of SP strip data will be only $3-4 \mathrm{~km}$ at the equator by the end of the mission, and shorter at higher latitudes.

\subsection{Orbit plane change operation}

Although the SELENE orbit interval between sequential revolutions will be about $100 \mathrm{~km}$ at the equator, the MI swath width is less than $20 \mathrm{~km}$, which is too narrow to have any overlap in sequential orbits at lower latitudes. Therefore, SELENE will change its orbital plane three times to achieve seamless MI global mapping in the nominal mission period: once for middle latitude observations and twice for lower latitude observations. Our proposal for the SELENE orbiter to execute necessary orbital plane change operations during the nominal mission period has been discussed and approved by the SELENE project.

\subsection{Calibration operation}

Raw TC, MI, and SP data contain dark-level outputs that were set in the flight model development phase. The dark levels are varied with dark currents depending on temperature and radiation damage conditions. We acquired darklevel data and its dependence on temperature in the prelaunch test phase to remove the dark level and to derive the net values for reflected light from the lunar surface. However, the dark levels and their dependence on temperature might be changed because of radiation damage or other factors. Therefore, we will regularly acquire dark-level data to carry out night-side operations. It will be activated for SP before the terminator passes from the night side to the day side, and will be deactivated after the terminator passes from the day side to the night side to collect dark level data. The straight imaging operations of the SP might increase the data volume for MI and TC, which will not be allowed. We will execute an independent operation sequence to acquire dark-level data in the night side at least once per revolution for MI and several times a week for TC.

We collected pre-flight calibration data using wellcalibrated integrating spheres to calibrate the radiance. However, calibration coefficients might change for various reasons, such as structural transformation, contamination, or radiation damage during the launch and in the space environment. For that reason, in-flight radiometric calibrations, particularly for MI and SP, are necessary to acquire the corrected calibration coefficients. The lunar surface is a good calibration site because it has no atmosphere and no ocean or plants that might drastically change the spectral reflectance. Some areas on the Moon that were found to be homogeneous and substantial in ground-based observations or previous lunar explorations have been proposed as sites. Mare Serenitatis and the Apollo 16 landing site $\left(6^{\circ} \mathrm{S}\right.$, $\left.15.5^{\circ} \mathrm{E}\right)$ are particularly regarded as appropriate reference sites because the mineral abundances in those areas have been estimated using returned samples. We have chosen a large area (about $8 \mathrm{~km}$ in the east-west direction) near the Apollo 16 landing site as the reference site. Both SP and MI will likely miss observations of the reference site if we do not execute any intentional pointing operations; therefore, SELENE will change its attitude around a role axis using the momentum wheel so that MI and SP can observe the site three times during the one-year mission period (see Fig. 7).

The SP will also calibrate the data using onboard wavelength/radiance calibration lamps. The calibration lamp operation will be executed several times a week.

\subsection{Extra observations}

The lunar surface exhibits various features: dark or bright and flat, uneven, or steep. Consequently, it might be difficult to acquire data for entire areas with one set of operation modes of the exposure time (for TC, MI, and SP), compression table (for TC), and resolution (for SP). Therefore, we plan to execute extra observations for important and interesting areas with a set of appropriate observation modes when the allowed observable data volumes are increased. The candidates for extra observation target areas will be listed before the SELENE launch. The lists of candidates for extra observation will be updated at monthly LISM science team meetings by referring to past observation results and the future operation plans of the SELENE orbiter and other mission instruments.

\section{Conclusion and Discussion}

An optical instrument, the Lunar Imager/Spectrometer (LISM), will be installed on the Japanese 2-ton class SELENE lunar orbiter, which will be launched in the summer of 2007. The scientific requirements of LISM are to provide (1) high-resolution data at $10 \mathrm{~m}$ spatial resolution in appropriate solar elevation angle conditions less than 30$40^{\circ}$, (2) multi-band images with spatial resolution at $100 \mathrm{~m}$ or higher, (3) stereo-pair images to produce digital terrain models (DTMs) with relative height resolution of a few tens of meters or better, (4) spectral profiling data in the visibleto-near-infrared range with onboard calibrators for wavelength and radiance. To satisfy those requirements, LISM consists of three sub-systems: a terrain camera (TC), which is a stereo-camera with $10 \mathrm{~m}$ resolution and a stereo angle of $30^{\circ}$, a multi-band imager (MI), which is a multicolor imager with four and five color bands with $20 \mathrm{~m} / 60 \mathrm{~m}$ spatial resolution in the visible and near-infrared regions, and a spectral profiler (SP), which is a line spectral profiler with a 400-mwide footprint and 300 spectral bands with 6-8 nm spectral resolution in the visible to near-infrared range. We have an LISM annual observation plan. Both the TC and MI will execute global mapping; the TC will perform high-contrast monoscopic mapping in two Moon cycles and stereoscopic mapping in three Moon cycles; the MI will perform multicolor mapping in six Moon cycles. SELENE's orbital plane will be changed three times during the mission period for MI global mapping. The TC and MI will compress their data on board to reduce the total volume provided. In addition, TC/MI observations will usually be divided into two compression rates for one SELENE revolution around the Moon, i.e. nominal compression rate observations and SP support observations, with a high compression rate to detect SP observation lines by one telescope system (TC or MI of one band). The SP will be operated in the daytime during the nominal mission. The east-west interval of SP strip data will be $3-4 \mathrm{~km}$ at the equator by the end of the mission, and shorter at higher latitudes. In conclusion, LISM will 


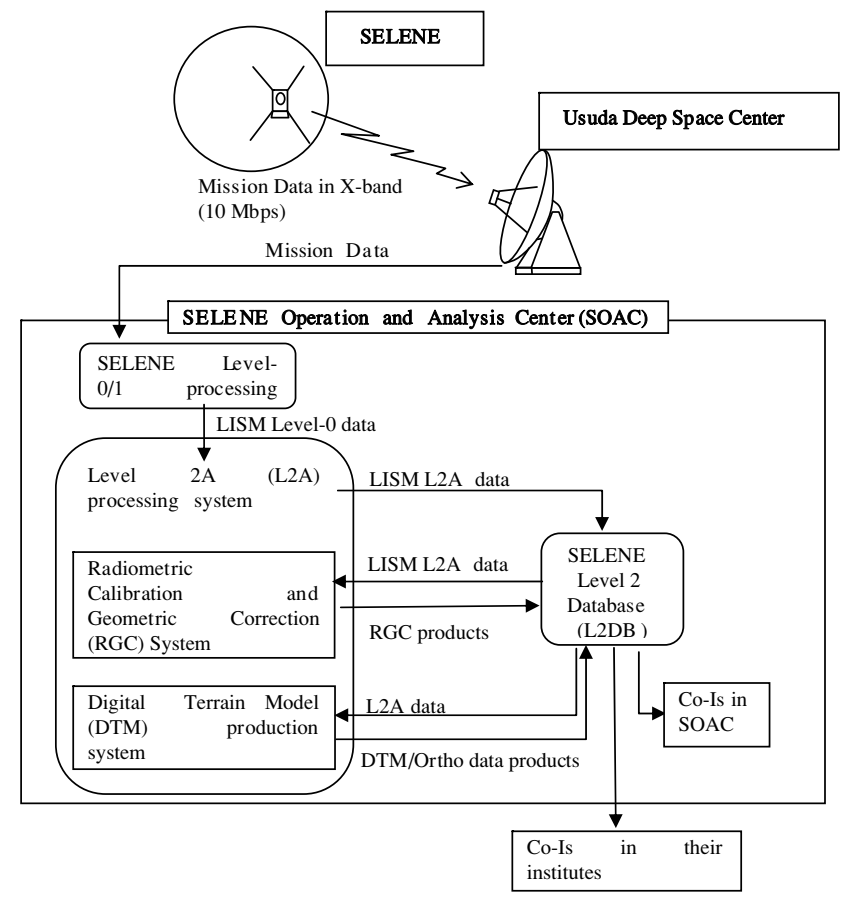

Fig. 10. The LISM mission data flow among the SELENE, Usuda Deep Space Center, SELENE Operation and Analysis Center, and the Co-Is in their institutes. The LISM data processing systems in the SOAC consists of three sub-systems: the Level $2 \mathrm{~A}$ processing system, the radiometric calibration and geometric correction system, and the digital terrain models (DTM) production system. The LISM products will be public via Level 2 database one year after the end of the nominal mission period.

provide two high-contrast images, stereo pair images, nineband color images, and a spectral strip of $4 \mathrm{~km}$ or shorter in the east-west interval. Furthermore, LISM will provide a fundamental dataset to advance lunar sciences and investigations of future human activities on the Moon.

The LISM flight model has been fabricated and tested to yield satisfactory performance. The final steps of preparation prior to the SELENE/LISM launch are to complete development of 1) operation support systems for busy weekly operational command planning and 2) ground-processing systems. The command timeline will be transferred from the ground station to the SELENE once a week. The LISM command sequence will differ daily to acquire data by appropriate observation modes. In addition, the regions that are missed because of operational problems with the spacecraft or ground station, lower altitude conditions in highland observations, unexpectedly excessive or minimal reflectance conditions, or other factors, must be compensated in subsequent opportunities during the mission period. Weekly operation planning would be too complex. Therefore, we are developing an operation support tool to aid the LISM operation team in systematically checking previous acquisition conditions and designing an efficient set of operational commands by verifying the command rules, the data rate by referring to the predicted compression rate and total volume per day, the appropriate exposure mode, and other elements. Three LISM data-processing systems will have been constructed in the SELENE Operation and Analysis Center (SOAC) at the Institute of Space and Astronautical
Science (ISAS). These systems include a Level 2A processing system that will produce the LISM raw data and attach necessary information for subsequent procedures, a radiometric calibration and geometric correction system to produce radiance and reflectance data with corrected geometry used to view the pointing vectors that are first obtained in pre-flight tests and later corrected using in-flight data (some of which will be mosaicked to serve as a source for geological base maps), and a digital terrain model (DTM) production system that produces DTMs and ortho image data from the TC stereo pairs (Demura et al., 2001; Haruyama et al., 2006b). Figure 10 depicts the LISM mission data flow. The primary LISM products, such as radiometrically calibrated and geometrically corrected radiance data, surface albedo or reflectance maps, and digital terrain models with ortho image data, produced using the systems will be stored in the SELENE Level 2 database. The LISM data will be analyzed by more than 20 LISM team members, who will acquire the information via the database and analyze data at their institutes. We plan to begin releasing carefully checked LISM data one year after the end of the nominal mission period, which starts in 2007, or by the beginning of 2010 to contribute to lunar and planetary sciences.

\section{References}

Basilevsky, A. T., H. U. Keller, A. Nathues, U. Mall, H. Hiesinger, and M. Rosiek, Scientific Objectives and Selection of Targets for the SMART1 Infrared Spectrometer (SIR), Planet. Space Sci., 52, 14, 1261-1285, 2004.

Butler, B. J., The Migration of Volatiles on the Surfaces of Mercury and the Moon, J. Geophys. Res., 102(E8), 19283-12291, 1997.

Demura, H., N. Hirata, H. Otake, M. Ohtake, A. Sugihara, M. Higa, T. Matsunaga, and J. Haruyama, Data Processing Flow and Products of LISM: Lunar Imager and SpectroMeter, 32nd Lunar Planet. Sci. Conf., abst. \#11648, 2001.

Feldman, W. C., A. B. Binder, B. L. Barraclough, and R. D. Belian, First results from the Lunar Prospector Spectrometers, 29th Lunar Planet. Sci. Conf., abst. \#1936, 1998.

Feldman, W. C., D. J. Lawrence, S. Maurice, R. C. Elphic, B. L. Barraclough, A. B. Binder, and P. G. Lucey, Classifi cation of Lunar Terranes using Neutron and Thorium Gamma-ray Data, 30th Lunar Planet. Sci. Conf., abst. \#2056, 1999.

Feldman, W. C., S. Maurice, D. J. Lawrence, R. C. Little, S. L. Lawson, O. Gasnault, R. C. Wiens, B. L. Barraclough, R. C. Elphic, T. H. Prettyman, J. T. Steinberg, and A. B. Binder, Evidence for Water Ice Near the Lunar Poles, J. Geophys. Res., 106(E10), 23,231-23,251, 2001.

Giguere, T. A., B. R. Hawke, L. R. Gaddis, D. T. Blewett, J. J. GillisDavis, P. G. Lucey, G. A. Smith, P. D. Spudis, and G. J. Taylor, Remote Sensing Studies of the Dionysius Region of the Moon, J. Geophys. Res., 111(E6), doi:10.1029/2005JE002639, 2006.

Hapke, B., Theory of Reflectance and Emittance Spectroscopy, 262, Cambridge Univ. Press, 1993.

Haruyama, J., H. Otake, M. Ohtake, A. Shiraishi, N. Hirata, and T. Matsunaga, LISM (Lunar Imager/SpectroMeter) Mission for SELENE Project, 31st Lunar Planet. Sci. Conf., abst. \#1317, 2000.

Haruyama, J., H. Otake, and M. Ohtake, LISM Mission for SELENE Project, Proc. 22nd Int. Sym. Tec. Space Sci., 1686-1691, 2001.

Haruyama, J., M. Ohtake, N. Hirata, R. Nakamura, and T. Matsunaga, Expected Performance of Lunar Imager/SpectroMeter on SELENE, 34th Lunar Planet. Sci. Conf., abst. \#1565, 2003a.

Haruyama, J., M. Ohtake, T. Matsunaga, N. Hirata, and LISM Working Group, Flight Model Performance of SELENE Terrain Camera, Proc. 23rd. Int. Sym. Tec. Space Sci., 1992-1996, 2003 b.

Haruyama, J., M. Ohtake, T. Matsunaga, and LISM Working Group, The Detectability of Degradation of Lunar Impact Crater by SELENE Terrain Camera, 35th Lunar Planet. Sci. Conf., abst. \#1496, 2004.

Haruyama, J., M. Ohtake, N. Hirata, R. Nakamura, and T. Matsunaga, Flight model performance of SELENE Terrain Camera (II), Proc. 25th Int. Sym. Tec. Space Sci., 857-862, 2005. 
Haruyama, J., M. Ohtake, T. Matsunaga, T. Morota, A. Yoshizawa, and LISM Working Group, Planned Digital Terrain Model Products from SELENE Terrain Camera Data, 37th Lunar Planet. Sci. Conf., abst. \#1132, 2006a.

Haruyama, J., M. Ohtake, T. Matsunaga, and LISM Working Group, Global High-resolution stereo mapping of the Moon with the SELENE Terrain Camera, in Adv. Geosci., 3: Planetary Science, World Scientific Publishing, edited by W.-H. Ip and A. Bhardwaj, pp. 101-108, $2006 \mathrm{~b}$.

Haruyama, J., T. Matsunaga, T. Morota, C. Honda, M. Torii, Y. Yokota, H. Kawasaki, M. Ohtake, and LISM Working Group, Pre-Launch Operation Planning of Lunar Imager/Spectrometer (LISM) on SELENE, 38th Lunar Planet. Sci. Conf., abst. \#1136, 2007.

Head, J. W., Lunar volcanism in space and time, Rev. Geophys. Space Phys., 14, 265-300, 1976.

Hörz, F., R. A. Grieve, G. D. Heiken, P. D. Spudis, and A. B. Binder, Lunar Surface, Processes, in The Lunar Sourcebook: A User's Guide to the Moon, edited by G. D. Heiken, D. Vaniman, and B. M. French, Lunar and Planet. Inst. and Cambridge Univ. Press, pp. 61-120, 1991.

Hiesinger, H. and J. M. Head, New Views of Lunar Geosiceces: An Introduction and Overview, in New Views of the Moon, Rev. Min. Gechem., 60, 1-81, 2006.

Hiesinger, H., R. Jaumann, G. Neukum, J. W. Head, and U. Wolf, Ages and Stratigraphy of Mare Basalts on the Lunar Nearside, J. Geophys. Res., 105(E12), 29239-29275, 2000.

Hiesinger, H., J. W. Head, U. Wolf, R. Jaumann, and G. Neukum, Ages and Stratigraphy of Mare B Basalts in Oceanus Procellarum, Mare Nubium, Mare Cognitum, and Mare Insularum, J. Geophys. Res., 108(E7), 5065, doi:10.1029/2002JE001985, 2003.

Iwata, T., N. Namiki, H. Hanada, H. Minamino, T. Takano, N. Kawano, K. Matsumoto, and S. Sasaki, SELENE Small Sub-Satellites for Lunar Gravity Observation, 38th Lunar Planet. Sci. Conf., abst. \#1557, 2007.

Jolliff, B. L., J. J. Gillis, L. A. Haskin, R. L. Korotev, and M. A. Wieczorek, Major Lunar Crustal Terrains: Surface Expression and Crust-mantle Origins, J. Geophys. Res., 105(E2), 4197-4216, 2001.

Kato, M., Y. Takizawa, and S. Sasaki, Selene Project Team, The SELENE Mission: Present Status and Science Goals, 38th Lunar Planet. Sci. Conf., abst. \#1211, 2007.

Lawrence, D. J., W. C. Feldman, R. C. Elphic, J. J. Hagerty, S. Maurice, G. W. McKinney, and T. H. Prettyman, Improved Modeling of Lunar Prospector Neutron Spectrometer Data: Implications for Hydrogen Deposits at the Lunar Poles, J. Geophys. Res., 111, E08001, doi:10.1029/2005JE002637, 2006.

Matsunaga, T., M. Ohtake, Y. Makiko, and J. Haruyama, Development of a Visible and Near-infrared Spectrometer for Selenological and Engineering Explorer (SELENE), in Hyperspectral Remote Sensing of the Land and Atmosphere, edited by W. L. Smith and Y. Yasuoka, Proc. SPIE, 4151, 32-39, 2001

Matsunaga, T., M. Ohtake, J. Haruyama, T. Sugihara, and LISM Work- ing Group, Environmental Test Resutls and Their Implications to the Perfommance of SELENE Spectral Profiler, Proc. 23rd Int. Sym. Tec. Space Sci., 1922-1927, 2003.

Melosh, H. J., Impact Cratering: A Geological Process, Oxford University Press, 1989.

Namiki, N., H. Hanada, T. Tsubokawa, N. Kawano, M. Ooe, K. Heki, T. Iwata, M. Ogawa, and T. Takano, Selenodetic Experiments of SELENE: Relay Subsatellite, Differential VLBI, and Laser Altimeter, Adv. Space Res., 23, 11, 1817-1820, 1999.

Neukum, G., B. A. Ivanov, and W. K. Hartmann, Cratering records in the inner solar system in relation to the lunar reference system, Space Sci. Rev., 96, 55-86, 2001.

Nozette, S. and the Clementine team, The Clementine Mission to the Moon: Scientific Overview, Science, 266, 1835-1839, 1994.

Nyquist, L. E. and C.-Y. Shih, The isotopic record of lunar volcanism, Geochim. Cosmochim. Acta, 56, 2213-2234, 1992.

Ohtake, M., J. Haruyama, and T. Matsunaga, Scientific Goals and Performance of Multiband Imager for the SELENE mission, Proc. 23rd Int. Sym. Tec. Space Sci., 1997-2000, 2003.

Ohtake, M., J. Haruyama, T. Matsunaga, T. Morota, Y. Yokota, C. Honda, A. Yamamoto, T. Arai, and H. Takeda, Objectives of the SELENE Multiband Imager and Spectral Study of Dho489, 38th Lunar Planet. Sci. Conf., abst. \#1829, 2007.

Pieters, C. M., Compositional diversity and stratigraphy of the lunar crust derived from reflectance spectroscopy, in Remote Geochemical Analysis Elemental and Mineralogical Composition, edited by C. M. Pieters and P. A. J. Englert, Cambridge University Press, Cambridge, pp. 309-339, 1993.

Pieters, C. M., L. Gaddis, B. Jolliff, and M. Duke, Rock Types of South Pole-Aitken Basin and Extent of Basaltic Volcanism, J. Geophys. Res., 106(E11), 28001-28022, 2001.

Sasaki, S., Y. Iijima, K. Tanaka, M. Kato, M. Hashimoto, H. Mizutani, and Y. Takizawa, The SELENE mission: Goals and status, Adv. Space Res., 31(11), 2335-2340, 2003.

Starukhina, L. V., Polar Regions of the Moon as a Potential Repository of Solar-Wind-Implanted Gases, Adv. Space Res., 37(1), 50-58, 2006.

Starukhina, L. V. and Y. G. Shkuratov, The lunar poles: water ice or chemically trapped hydrogen, Icarus, 147, 585-587, 2000.

Tompkins, S. and C. M. Pieters, Mineralogy of the Lunar Crust: Results from Clementine, Meteorit. Planet. Sci., 34(1), 25-41, 1999.

Wilhelms, D. E., The Geologic History of the Moon, U.S. Geol. Surv. Prof. Pap., 1348, 302 pp, 1987.

J. Haruyama (e-mail: haruyama.junichi@jaxa.jp), T. Matsunaga, M. Ohtake, T. Morota, C. Honda, Y. Yokota, M. Torii, Y. Ogawa, and the LISM Working Group 